PHYSICAL REVIEW D 89, 061502(R) (2014)

\title{
Effective-one-body model for black-hole binaries with generic mass ratios and spins
}

\author{
Andrea Taracchini, ${ }^{1}$ Alessandra Buonanno, ${ }^{1}$ Yi Pan, ${ }^{1}$ Tanja Hinderer, ${ }^{1}$ Michael Boyle, ${ }^{2}$ Daniel A. Hemberger, ${ }^{2,3}$ \\ Lawrence E. Kidder, ${ }^{2}$ Geoffrey Lovelace, ${ }^{4,3}$ Abdul H. Mroué, ${ }^{5}$ Harald P. Pfeiffer, ${ }^{5,6}$ Mark A. Scheel, ${ }^{3}$ \\ Béla Szilágyi, ${ }^{3}$ Nicholas W. Taylor, ${ }^{3}$ and Anil Zenginoglu ${ }^{3}$ \\ ${ }^{1}$ Department of Physics, Maryland Center for Fundamental Physics and Joint Space-Science Institute, \\ University of Maryland, College Park, Maryland 20742, USA \\ ${ }^{2}$ Center for Radiophysics and Space Research, Cornell University, Ithaca, New York 14853, USA \\ ${ }^{3}$ Theoretical Astrophysics 350-17, California Institute of Technology, Pasadena, California 91125, USA \\ ${ }^{4}$ Gravitational Wave Physics and Astronomy Center, California State University Fullerton, \\ Fullerton, California 92831, USA \\ ${ }^{5}$ Canadian Institute for Theoretical Astrophysics, 60 St. George Street, University of Toronto, \\ Toronto, Ontario M5S 3H8, Canada \\ ${ }^{6}$ Canadian Institute for Advanced Research, 180 Dundas Street West, Toronto, Ontario M5 G 1Z8, Canada
}

(Received 12 November 2013; published 13 March 2014)

Gravitational waves emitted by black-hole binary systems have the highest signal-to-noise ratio in LIGO and Virgo detectors when black-hole spins are aligned with the orbital angular momentum and extremal. For such systems, we extend the effective-one-body inspiral-merger-ringdown waveforms to generic mass ratios and spins calibrating them to 38 numerical-relativity nonprecessing waveforms produced by the SXS Collaboration. The numerical-relativity simulations span mass ratios from 1 to 8 , spin magnitudes up to $98 \%$ of extremality, and last for 40 to 60 gravitational-wave cycles. When the total mass of the binary is between 20 and $200 M_{\odot}$, the effective-one-body nonprecessing (dominant mode) waveforms have overlap above $99 \%$ (using the advanced-LIGO design noise spectral density) with all of the 38 nonprecessing numerical waveforms, when maximizing only on initial phase and time. This implies a negligible loss in event rate due to modeling. We also show that - without further calibration - the precessing effective-onebody (dominant mode) waveforms have overlap above 97\% with two very long, strongly precessing numerical-relativity waveforms, when maximizing only on the initial phase and time.

DOI: $10.1103 /$ PhysRevD.89.061502

\section{INTRODUCTION}

In the next few years, second-generation ground-based interferometers, such as advanced LIGO [1], advanced Virgo [2], and KAGRA [3], will start to collect data with unprecedented sensitivity, making the long-sought detection of gravitational waves (GWs) a realistic prospect. Coalescing binaries of compact objects are among the most promising astrophysical sources in the accessible frequency band of such experiments. The search for GWs from these sources exploits the matched-filtering technique, in which the noisy output of the interferometer is correlated with a bank of template waveforms describing all expected signals. An accurate knowledge of the gravitational radiation is thus crucial for maximizing the chances of detection. However, matched filtering not only requires templates that are accurate, but their generation must also be sufficiently cheap that they cover the entire physical parameter space. While in principle the most precise waveforms are obtained by solving Einstein's equations in numerical relativity (NR), their considerable computational cost makes it necessary to resort to analytical models that meet both criteria of accuracy and computational efficiency.

A unified analytical description of the entire compact binary coalescence, from the quasicircular inspiral, through
PACS numbers: 04.25.D-, 04.25.dg, 04.25.Nx, 04.30.-w

the merger, and to the ringdown (RD) of the remnant, is achieved by the effective-one-body (EOB) model [4]. In the EOB approach, one replaces the real problem of two compact objects of mass $m_{i}$, spin $S_{i}(i=1,2)$, and mass ratio $q$ orbiting each other with the effective problem of an extreme mass-ratio binary, where the more massive object is a deformed-Kerr black hole (BH) and the small object is an effective spinning particle. The deformation parameter of the Kerr metric is the symmetric mass ratio $\nu \equiv q /(1+q)^{2}$. The EOB model incorporates results from post-Newtonian (PN) theory (in resummed form), BH perturbation theory, and more recently also from the gravitational self-force formalism. A mapping between the physical parameters of the two problems is established by requiring that the effective dynamics is equivalent (when PN expanded in powers of $1 / c^{2}$ ) to the original, PN-expanded dynamics. Thus, solving exactly the effective problem of a spinning particle in the deformed-Kerr geometry amounts to introducing a particular nonperturbative method for resumming the PN-expanded equations of motion.

The accuracy of the EOB waveforms has recently been improved by including in the EOB dynamics higher-order (yet unknown) PN terms and calibrating them to NR simulations, which have progressively grown in number, 
length, and accuracy. State-of-the-art calibrations of these adjustable parameters in the nonspinning sector (including also higher harmonics) can be found in Refs. [5-7]. An EOB model for spinning, nonprecessing $\mathrm{BH}$ binaries was calibrated to five nonspinning and only two spinning, nonprecessing NR simulations in Ref. [6] ${ }^{1}$; it can generate dominant $(2,2)$ mode waveforms for any mass ratio, but only for $\mathrm{BH}$ spin magnitudes up to 0.6. Moreover, the EOB model in Ref. [6] was compared and validated against a large set of new NR simulations of nonprecessing BHs produced by several groups within the numerical-relativity and analytical-relativity (NRAR) Collaboration [8]. Recently, Ref. [9] provided a general procedure to generate EOB waveforms for spinning, precessing $\mathrm{BH}$ binaries starting from a generic spinning, nonprecessing EOB model; when using the EOB model in Ref. [6] as the underlying nonprecessing model, the authors found remarkable agreement with two precessing NR simulations. Finally, the conservative dynamics of the EOB model has also been tested and validated through the study of the periastron advance in $\mathrm{BH}$ binaries [10].

In this work, we calibrate the nonprecessing sector of a generic spinning EOB model to the $(2,2)$ mode of a catalog of highly accurate NR simulations produced by the SXS Collaboration [13,14]. They include eight nonspinning and 30 spinning, nonprecessing BH binaries with spins up to $98 \%$ of extremality, they cover mass ratios up to 8 , and have orbital eccentricities in the range of a few percent down to $10^{-5}$. The simulations follow more orbits on average (up to 35.5), allowing a more reliable calibration of analytical waveforms.

\section{EFFECTIVE-ONE-BODY MODEL}

In what follows we set $G=c=1$. Let $\hat{\boldsymbol{L}}$ be the direction perpendicular to the binary's instantaneous orbital plane, and let us define the dimensionless projections of the spins along $\hat{\boldsymbol{L}}$ as $\chi_{i}=\left(\boldsymbol{S}_{i} \cdot \hat{\boldsymbol{L}}\right) / m_{i}^{2}$. We assume $m_{1} \geq m_{2}$, hence $q \equiv m_{1} / m_{2} \geq 1$. In the spinning EOB formalism of Ref. [15], the effective Hamiltonian $H_{\text {eff }}$ is that of a particle of mass $\mu \equiv m_{1} m_{2} /\left(m_{1}+m_{2}\right)$ and effective spin $\boldsymbol{S}^{*} \equiv$ $\boldsymbol{S}^{*}\left(\boldsymbol{S}_{1}, \boldsymbol{S}_{2}\right)$ moving in a deformed-Kerr geometry of mass $M \equiv m_{1}+m_{2}$ and spin $\boldsymbol{S}_{\mathrm{Kerr}} \equiv \boldsymbol{S}_{1}+\boldsymbol{S}_{2}$; the conservative orbital dynamics is then derived via Hamilton's equations using the real EOB-resummed Hamiltonian

$$
H_{\text {real }}=M \sqrt{1+2 \nu\left(\frac{H_{\text {eff }}}{\mu}-1\right)}-M .
$$

We use here the same EOB Hamiltonian as in Ref. [6], but augment the deformed-Kerr metric potential $\Delta_{u}$ with 4PN nonspinning terms to obtain [15]

\footnotetext{
${ }^{1}$ The EOB models of Refs. [5,6,11] have been implemented in the LIGO Algorithm Library under the names EOBNRv1, EOBNRv2, and SEOBNRv1, respectively, and have been used in $\mathrm{GW}$ searches [12].
}

$\Delta_{u}(u)=\bar{\Delta}_{u}(u)\left[1+\nu \Delta_{0}+\nu \log \left(1+\sum_{i=1}^{5} \Delta_{i} u^{i}\right)\right]$

where $u \equiv 1 / r$ and $r$ is the EOB radial coordinate in units of $M$. Here,

$$
\begin{gathered}
\bar{\Delta}_{u}(u)=\chi_{\mathrm{Kerr}}^{2}\left(u-\frac{1}{r_{+}^{\mathrm{EOB}}}\right)\left(u-\frac{1}{r_{-}^{\mathrm{EOB}}}\right), \\
r_{ \pm}^{\mathrm{EOB}}=\left[1 \pm\left(1-\chi_{\mathrm{Kerr}}^{2}\right)^{1 / 2}\right](1-K \nu),
\end{gathered}
$$

with $\chi_{\text {Kerr }} \equiv\left(S_{\text {Kerr }} \cdot \hat{\boldsymbol{L}}\right) / M^{2}$; the coefficients $\Delta_{0}, \ldots, \Delta_{5}$ are determined by requiring that $\Delta_{u}$ agrees with the Taylorexpanded EOB potential $A(r)[16,17]$ up to $4 \mathrm{PN}$ order. By construction, $r_{ \pm}^{\mathrm{EOB}}$ reproduce the Kerr horizons when $\nu=0$. Similar to what was done in Ref. [6], we exploit $K$ as an adjustable parameter, i.e., a parameter that we calibrate to NR waveforms. For the identification between the effective particle's spin $S^{*}$ and the spins $S_{i}$ we use the 3.5PN-accurate spin mapping of Ref. [15], with all the arbitrary gauge parameters set to zero and with the addition of a 4.5PN spin-orbit term of the form $\left(d_{\mathrm{SO}} \nu \boldsymbol{S}_{\mathrm{Kerr}}\right) / r^{3}$, where $d_{\mathrm{SO}}$ is an adjustable parameter. The EOB description of conservative spin effects is completed by adding a 3PN spin-spin term of the form $d_{\mathrm{SS}} \nu\left(\boldsymbol{S}_{1}^{2}+\boldsymbol{S}_{2}^{2}\right) / r^{4}$ to $H_{\mathrm{eff}} / \mu$, where $d_{\mathrm{SS}}$ is another adjustable parameter.

The adjustable parameters are chosen to be polynomials in $\nu$ whose coefficients are determined by minimizing the phase and relative amplitude difference between EOB and NR waveforms [defined as in Eqs. (29) and (30) of Ref. [6]] via the numerical simplex method for each mass ratio. First, we calibrate the nonspinning sector and find $K=$ $1.712-1.804 \nu-39.77 \nu^{2}+103.2 \nu^{3}$, where the $\nu$-independent term is consistent with the frequency shift of the innermost stable circular orbit (ISCO) due to conservative self-force effects in the small-mass-ratio limit [18]. Next, we calibrate the spin parameters and obtain $d_{\mathrm{SO}}=-74.71-156.0 \nu+$ $627.5 \nu^{2}$ and $d_{\mathrm{SS}}=8.127-154.2 \nu+830.8 \nu^{2}$.

Dissipative effects are modeled by supplementing Hamilton's equations with a radiation-reaction force which is a sum over (time derivatives of) the -2 -spin-weighted spherical modes at infinity. In our model, these modes are written as a factorized resummation of the PN waveforms $[19,20]$

$$
h_{\ell m}^{\mathrm{F}}=h_{\ell m}^{(N, \varepsilon)} \hat{S}_{\mathrm{eff}}^{(\varepsilon)} T_{\ell m} e^{i \delta_{\ell m}}\left(\rho_{\ell m}\right)^{\ell}
$$

(see Ref. [6] for the definition of the individual factors). In particular, here we also include comparable-mass spin-orbit and spin-spin effects up to $2 \mathrm{PN}$ order, using the most recent PN-waveform calculations in Ref. [21]. We use the $\rho_{\ell m}$ factorization in Eq. (4) for all modes except those with $\ell \leq 4$ and odd $m$, which instead follow the prescription of Ref. [6] [see the discussion above Eq. (A8a) therein]. 
In addition, we also include all the known spin effects from the test-particle limit given in Ref. [20], by replacing the Kerr spin parameter $a / M$ with $\chi_{\text {Kerr }}$; this helps the modeling of unequal-mass, spinning systems. As such, the mode amplitudes contain no adjustable parameters. In fact, the improved knowledge of the nonspinning sector (i.e., the addition of $4 \mathrm{PN}$ terms in $\Delta_{u}$ ) allowed us to remove the nonspinning adjustable parameter $\rho_{22}^{(4)}$ which had been introduced in Ref. [6], thus simplifying the nonspinning model. The resulting residuals on the amplitude of the $(2,2)$ mode are within a few percent at merger for $\chi_{1,2} \sim 1$ even without adding nonquasicircular (NQC) corrections. However, we need to introduce an adjustable parameter in the spin terms of the phase $\delta_{22}$ to enhance the EOB GW frequency close to merger with respect to its leading-order value (twice the orbital frequency $\Omega$ ), which tends to underestimate the NR value for $\partial_{t} \phi_{22}$ when spins are close to 1 . For $\chi_{1,2}=0.98$, we find that the ISCO is crossed only $10 M$ before the light ring crossing, thus greatly reducing the region in which the nonquasicircular corrections (see below) can be effective. Explicitly, if $\chi \geq 0$, we add the 3PN term $540 \nu \chi(M \Omega)^{2}$ to $\delta_{22}$, where $\chi \equiv \chi_{\mathrm{S}}+$ $\chi_{\mathrm{A}} \sqrt{1-4 \nu} /(1-2 \nu)$, with $\chi_{\mathrm{S}, \mathrm{A}} \equiv\left(\chi_{1} \pm \chi_{2}\right) / 2$.

NQC effects that become important near the merger are included in $h_{22}^{\mathrm{F}}$ through a factor $N_{22}$ [see Eq. (18) of Ref. [6]]. The NQC coefficients are fixed by requiring that the EOB $(2,2)$ mode agrees with the NR input values for $\left|h_{22}\right|, \partial_{t}\left|h_{22}\right|, \partial_{t}^{2}\left|h_{22}\right|, \partial_{t} \phi_{22}$, and $\partial_{t}^{2} \phi_{22}$ evaluated at the peak of $\left|h_{22}\right|$. Using the $38 \mathrm{NR}$ nonprecessing waveforms in the SXS catalog and Teukolsky waveforms computed in the small-mass-ratio limit [22], we updated the fitting formulas for the NR input values given in Table IV of Ref. [6]. We use these to iteratively compute the NQC coefficients as described in Sec. IIB of Ref. [6]. While previous nonspinning EOB models [11] were calibrated without enforcing any time delay between the peak in the $(2,2)$ amplitude and in the orbital frequency, here, as in Refs. [5,6], we require a lag $\Delta t_{\text {peak }}^{22}$ which varies with the physical parameters of the binary. The idea of introducing $\Delta t_{\text {peak }}^{22}$ into the spinning model was inspired by studies in the small-massratio limit, where such time delay was first seen with EOB trajectories sourcing Teukolsky waveforms [23] and accurately quantified in Ref. [22]. Finally, the inspiral-plunge waveform is simply defined as $h_{22}^{\text {insp-plunge }} \equiv N_{22} h_{22}^{\mathrm{F}}$, and $h_{\ell m}^{\text {insp-plunge }} \equiv h_{\ell m}^{\mathrm{F}}$ when $(\ell, m) \neq(2,2)$.

As usual, the EOB merger-RD waveform is built as a linear combination of quasi-normal-modes (QNMs) of the remnant $\mathrm{BH}[4]$

$$
h_{\ell m}^{\text {merger-RD }}(t)=\sum_{n=0}^{N-1} A_{\ell m n} e^{-i \sigma_{\ell m n}\left(t-t_{\text {match }}^{\ell m}\right)},
$$

where $N$ is the number of overtones, $t_{\text {match }}^{\ell m}$ is the time when $\left|h_{\ell m}^{\text {insp-plunge }}\right|$ peaks, $A_{\ell m n}$ is the complex amplitude of the $n$th overtone of the $(\ell, m)$ mode, and $\sigma_{\ell m n}=\omega_{\ell m n}-$ $i / \tau_{\ell m n}$ is its complex frequency having positive (real) frequency $\omega_{\ell m n}$ and decay time $\tau_{\ell m n}$. The frequencies $\sigma_{\ell m n}$ depend on the mass $M_{f}$ and spin $a_{f}$ of the final Kerr $\mathrm{BH}$, and are tabulated in Ref. [24]. To predict $M_{f}$ we use the phenomenological formula proposed by Ref. [25], but we replace its equal-mass limit [Eq. (11) therein] with the highly accurate fit given in Eq. (9) of Ref. [14]. To compute $a_{f}$, we start from the formula of Ref. [26] (which also predicts the direction of the final spin for precessing binaries), and use the simulations in the SXS catalog to refit its nonprecessing limit; the main change we introduce is four new fitting coefficients designed to improve the equal-mass, high-spin corner of the parameter space, where the prediction of Ref. [26] has residuals exceeding 5\%. We improve the stability of the ringdown modeling across the entire parameter space by (i) assuming a monotonic behavior of $a_{f}$ with decreasing $\nu$ for extremal spins, and (ii) replacing some of the higher physical overtones with pseudo-QNMs that depend on the merger frequency, on $\sigma_{220}$ and on $\nu$, and moderate the rise of the ringdown $\mathrm{GW}$ frequency $[5,6]$.

Finally, the complete inspiral-merger-ringdown waveform is built as the smooth matching of $h_{\ell m}^{\text {insp-plunge }}$ to $h_{\ell m}^{\text {merger-RD }}$ at $t_{\text {match }}^{\ell m}$, over an interval $\Delta t_{\text {match }}^{\ell m}$, following the hybrid matching procedure of Ref. [5] to fix the coefficients $A_{\ell m n}$ in Eq. (5).

\section{RESULTS AND DISCUSSION}

The SXS catalog includes eight nonspinning $\mathrm{BH}$ binaries with $q=1,1.5,2,3,4,5,6,8$, and 30 spinning, nonprecessing $\mathrm{BH}$ binaries with $q=1$ and $\chi_{1}=\chi_{2}=$ $0.98,0.97, \pm 0.95, \pm 0.9,0.85, \pm 0.8, \pm 0.6, \pm 0.44$, $\pm 0.2 ; q=1,1.5,3,5,8, \chi_{1}= \pm 0.5$, and $\chi_{2}=0 ; q=1.5$ and $\chi_{1}=-\chi_{2}= \pm 0.5 ; q=2, \chi_{1}=0.6$, and $\chi_{2}=0 ; q=3$ and $\chi_{1}=\chi_{2}= \pm 0.5$. We find that to accurately match all 38 nonprecessing waveforms, it is sufficient to calibrate the EOB model to a much smaller subset of them. However, since our goal is an accurate model for the entire parameter space, most of which is not covered by the NR waveforms, we prefer to exploit all available nondegenerate NR information in the calibration. In Fig. 1 we compare the EOB waveforms to all the 38 nonprecessing NR waveforms by computing their unfaithfulness

$$
\bar{F} \equiv 1-\max _{t_{0}, \phi_{0}} \frac{\left\langle h_{22}^{\mathrm{EOB}}, h_{22}^{\mathrm{NR}}\right\rangle}{\left\|h_{22}^{\mathrm{EO}}|| \mid h_{22}^{\mathrm{NR}}\right\|},
$$

where $t_{0}$ and $\phi_{0}$ are the initial time and phase, $\|h\| \equiv \sqrt{\langle h, h\rangle}$, and the inner product between two waveforms is defined as $\left\langle h_{1}, h_{2}\right\rangle \equiv 4 \operatorname{Re} \int_{f_{\min }}^{\infty} \tilde{h}_{1}(f) \tilde{h}_{2}^{*}(f) /$ $S_{n}(f) d f$, where $S_{n}(f)$ is the zero-detuned, high-power noise spectral density of advanced LIGO [1] and $f_{\min }$ is the starting frequency of the NR waveform (after junk radiation has settled). The normalized inner product of two waveforms is their overlap. We do not hybridize the NR waveforms at low frequency $\left(f<f_{\min }\right)$ with any analytic 
ANDREA TARACCHINI

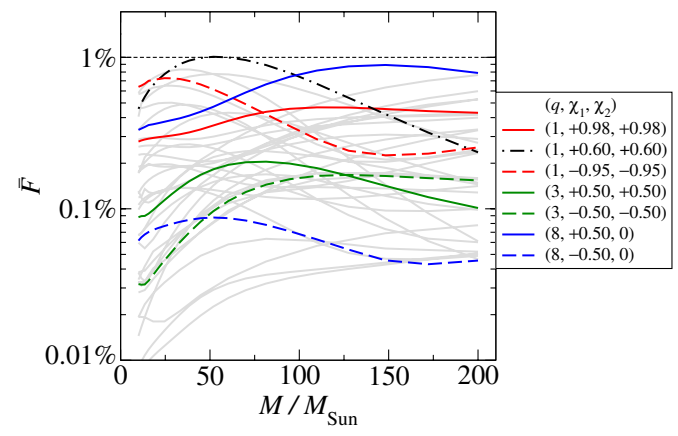

FIG. 1 (color online). Unfaithfulness of $(2,2)$ EOB waveforms for all the 38 nonprecessing BH binaries in the SXS catalog. Only a few selected cases are labeled in the legend.

approximant but instead taper the EOB waveforms. When $M \lesssim 100 M_{\odot}$ the NR waveforms do not cover the entire frequency bandwidth of the detector, but we expect that the unfaithfulness $\bar{F}$ would not change much when longer NR waveforms will be employed because the EOB calibration has been shown to be quite stable with respect to the number of GW cycles used for the calibration [27]. The unfaithfulness is always below $1 \%$ for total masses from 20 to $200 M_{\odot}$, implying a negligible loss in event rate due to the modeling error alone.

To estimate the NR error for each binary configuration, we choose the NR simulation with the largest number of cycles, with the highest resolution, and extrapolated to infinity with extrapolation order $N=3$ as the fiducial waveform. We then compute the model's unfaithfulness against NR waveforms (i) with a different extrapolation order but the same resolution and (ii) with a different resolution but the same extrapolation order, and obtain a conservative error bound on $\bar{F}$ from the difference between the fiducial and the most discrepant waveform. For the binary with $q=1$ and $\chi_{1}=\chi_{2}=0.98$, which we take as a representative case for the rest of the catalog, the errors on $\bar{F}$ are within $0.005 \%$.

Figure 2 shows the agreement between EOB and NR waveforms for the nearly extremal BH binary with $q=1$ and $\chi_{1}=\chi_{2}=0.98$, when aligning them at their amplitude peak; the phase difference is always within $0.6 \mathrm{rad}$. The coordinate invariant relation $\hat{E}(\hat{J})$ between the specific energy $\hat{E}$ and the total angular momentum $\hat{J}$ is a useful tool for evaluating analytical descriptions of the binary dynamics [28]. In Fig. 3, for the cases with $q=1$ and $\chi_{1}=\chi_{2}=-0.95,0.98$, we compare $\hat{E}(\hat{J})$ from NR (using Cauchy-characteristics-extracted waveforms), the conservative uncalibrated EOB model, and the EOB model calibrated in this paper. The numerical errors of $\hat{E}(\hat{J})$ increase from $10^{-5}$ at low frequency to $10^{-4}$ at high frequency. We find that when the spins are close to extremal, there is a difference of $10^{-3}$ between NR and analytical (EOB or even PN) $\hat{E}(\hat{J})$ at low frequency that is not explained by numerical errors. By contrast the
PHYSICAL REVIEW D 89, 061502(R) (2014)
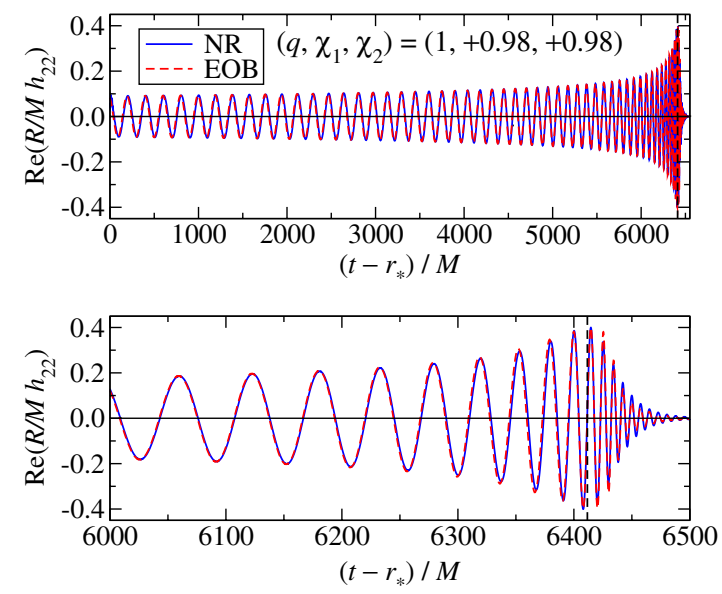

FIG. 2 (color online). $\quad \mathrm{NR}$ and EOB $(2,2)$ waveforms of the $\mathrm{BH}$ binary with $q=1$ and $\chi_{1}=\chi_{2}=0.98$. The two waveforms are aligned at their amplitude peak (marked by a vertical dashed line). $R$ is the distance to the source and $r_{*}$ is the tortoise coordinate.

difference is within numerical errors when the spin magnitudes are less than $\sim 0.6$. We plan to further investigate those results in the future. The cusps in the conservative EOB curves indicate the presence of an ISCO; this point lies $60 M(10 M)$ in time before merger for spin -0.95 (0.98). The calibrated EOB curves instead extend up to the light ring, which is very close to the merger. The good agreement between EOB and NR results validates the calibration procedure in yielding an accurate description of the binary evolution up to merger. The improved model for the nonprecessing limit developed here (as compared to Ref. [6]) is also the foundation for precessing binaries, via the procedure of transforming from the precessing frame to an inertial frame described in Ref. [9]. Without further
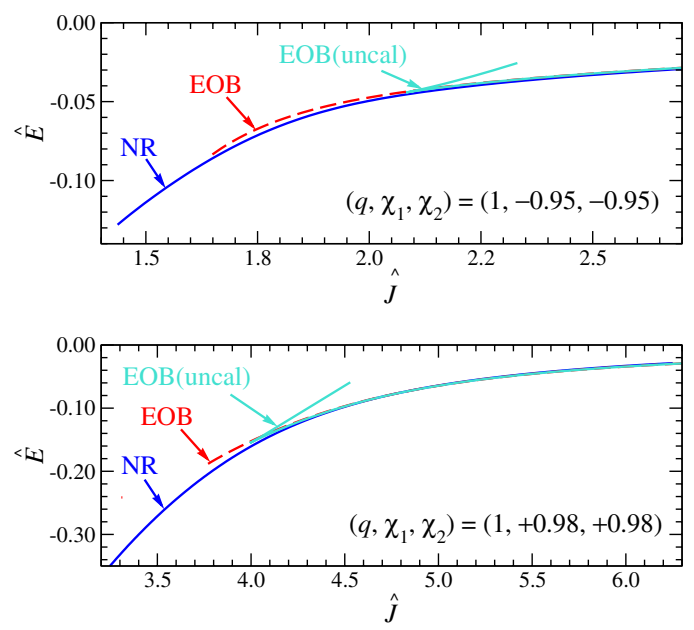

FIG. 3 (color online). The specific binding energy $\hat{E}=E / \mu$ as a function of the dimensionless total angular momentum $\hat{J}=$ $J /(\mu M)$ of the $\mathrm{BH}$ binaries with $q=1$ and $\chi_{1}=\chi_{2}=$ $-0.95,0.98$ computed in NR, conservative uncalibrated EOB model and the calibrated EOB model of this paper. 

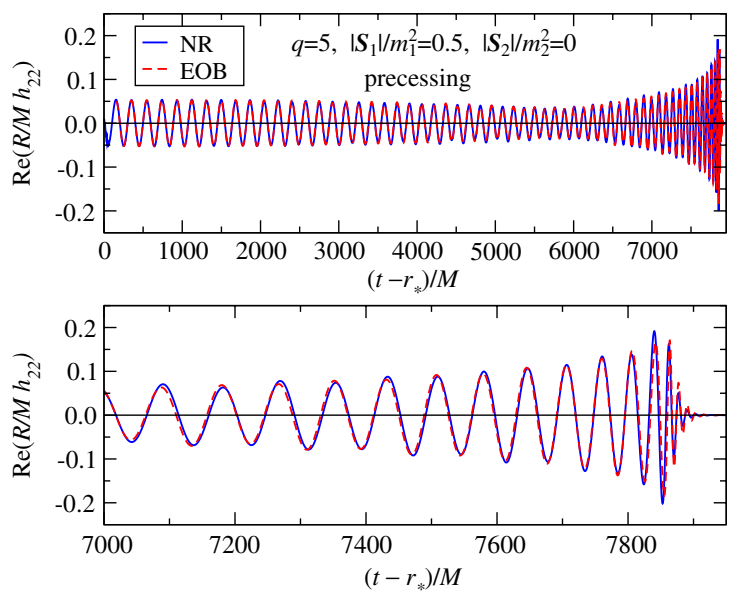

FIG. 4 (color online). NR and EOB $(2,2)$ precessing waveforms of the BH binary with $q=5$ and initial spins $\left|\boldsymbol{S}_{1}\right| / m_{1}^{2}=0.5$ in the orbital plane and $\left|\boldsymbol{S}_{2}\right| / m_{2}^{2}=0$. The two waveforms are aligned at low frequency. $R$ is the distance to the source and $r_{*}$ is the tortoise coordinate.

calibration, we tested our model against the two long precessing waveforms that were used in Ref. [9], one with $q=3$ and initial spins (both of magnitude 0.5 ) respectively in the orbital plane and antialigned with $\hat{\boldsymbol{L}}$, and the other with $q=5$ and initial spins (of magnitude 0.5 and 0 , respectively) in the orbital plane, and found that $\bar{F}<3 \%$ for both cases. We show EOB and NR precessing waveforms of the $q=5$ case in Fig. 4.

\section{CONCLUSIONS}

Using 38 NR $(2,2)$ mode waveforms for spinning, nonprecessing $\mathrm{BH}$ binaries produced by the SXS Collaboration, we have calibrated the nonprecessing sector of the EOB model of Refs. $[9,15]$, which is valid for any mass ratio and spin. Throughout the entire parameter space covered by the NR simulations, the EOB model of this paper achieves an unfaithfulness within $1 \%$, implying a negligible loss in event rate due to the modeling error alone. By extending the EOB model to nearly extremal spins, we have increased the distance reach of advanced detectors. Furthermore, the EOB model can be used to generate precessing waveforms using the prescriptions in Ref. [9]. The EOB model developed here will be implemented in the
LIGO Algorithm Library, so that it can be employed by advanced LIGO and Virgo to detect gravitational waves from spinning binary $\mathrm{BHs}$ and to extract physical information once the waves are observed. EOB models are computationally expensive to generate (although far faster than doing NR simulations) and work is under way to speed them up. Future work will continue to improve the EOB radiation-reaction sector and the calibration of the EOB conservative dynamics, extend the modeling to higherorder modes, investigate the performance of the model against the precessing configurations in the SXS catalog, and check its stability against much longer NR simulations, thus extending the studies recently carried out in Ref. [27] for nonspinning BHs.

\section{ACKNOWLEDGMENTS}

A. B., T. H., Y. P., and A. T. acknowledge partial support from NSF Grants No. PHY-0903631 and No. PHY1208881. A. B. also acknowledges partial support from NASA Grant No. NNX12AN10G. T. H. and A. T. also acknowledge support from the Maryland Center for Fundamental Physics. A. M. and H. P. gratefully acknowledge support from NSERC of Canada, the Canada Chairs Program, and the Canadian Institute for Advanced Research. M. B., D. H., and L. K. gratefully acknowledge support from the Sherman Fairchild Foundation, and from NSF Grants No. PHY-1306125 and No. PHY-1005426 at Cornell. M.S., B.S., N.T., and A.Z. acknowledge support from the Sherman Fairchild Foundation and from NSF Grants No. PHY-106881, No. PHY-1005655, and No. DMS-1065438 at Caltech. G. L. acknowledges partial support from NSF Grant No. PHY-1307489. Simulations used in this work were computed with the SPEC code [29]. Computations were performed on the Zwicky cluster at Caltech, which is supported by the Sherman Fairchild Foundation and by NSF Grant No. PHY-0960291, on the NSF XSEDE network under Grant No. TG-PHY990007N, on the Orca cluster supported by Cal State Fullerton, and on the GPC supercomputer at the SciNet HPC Consortium [30]. SciNet is funded by the Canada Foundation for Innovation under the auspices of Compute Canada, the Government of Ontario, Ontario Research Fund-Research Excellence, and the University of Toronto.
[1] D. Shoemaker (LIGO Collaboration), LIGO Report No. T0900288-v3, 2010.

[2] F. Acernese (Virgo Collaboration), Report No. vIR027A09, 2010.
[3] K. Somiya (KAGRA Collaboration), Classical Quantum Gravity 29, 124007 (2012).

[4] A. Buonanno and T. Damour, Phys. Rev. D 59, 084006 (1999); 


\section{ANDREA TARACCHINI}

[5] Y. Pan, A. Buonanno, M. Boyle, L. T. Buchman, L. E. Kidder, H. P. Pfeiffer, and M. A. Scheel, Phys. Rev. D 84, 124052 (2011).

[6] A. Taracchini, Y. Pan, A. Buonanno, E. Barausse, M. Boyle, T. Chu, G. Lovelace, H. P. Pfeiffer, and M. A. Scheel, Phys. Rev. D 86, 024011 (2012).

[7] T. Damour, A. Nagar, and S. Bernuzzi, Phys. Rev. D 87, 084035 (2013).

[8] I. Hinder, A. Buonanno et al. (NRAR Collaboration), Classical Quantum Gravity 31, 025012 (2013).

[9] Y. Pan et al., arXiv:1307.6232.

[10] A. Le Tiec, A.H. Mroué, L. Barack, A. Buonanno, H. P. Pfeiffer, N. Sago, and A. Taracchini, Phys. Rev. Lett. 107, 141101 (2011); T. Hinderer, A. Buonanno et al., Phys. Rev. D 88, 084005 (2013).

[11] A. Buonanno, Y. Pan, J. Baker, J. Centrella, B. Kelly, S. McWilliams, and J. van Meter, Phys. Rev. D 76, 104049 (2007).

[12] J. Aasi et al. (LIGO Scientific Collaboration and Virgo Collaboration), Phys. Rev. D 87, 022002 (2013).

[13] A. H. Mroue et al., Phys. Rev. Lett. 111, 241104 (2013); A. H. Mroue and H.P. Pfeiffer, arXiv:1210.2958; D. A. Hemberger, M. A. Scheel, L. E. Kidder, B. Szilágyi, G. Lovelace, N. W. Taylor, and S. A. Teukolsky, Classical Quantum Gravity 30, 115001 (2013).

[14] D. A. Hemberger, G. Lovelace, T. J. Loredo, L. E. Kidder, M. A. Scheel, B. Szilágyi, N. W. Taylor, and S. A. Teukolsky, Phys. Rev. D 88, 064014 (2013).
PHYSICAL REVIEW D 89, 061502(R) (2014)

[15] E. Barausse and A. Buonanno, Phys. Rev. D 84, 104027 (2011).

[16] E. Barausse, A. Buonanno, and A. Le Tiec, Phys. Rev. D 85, 064010 (2012).

[17] D. Bini and T. Damour, Phys. Rev. D 87, 121501 (2013).

[18] L. Barack and N. Sago, Phys. Rev. D 81, 084021 (2010).

[19] T. Damour, B. R. Iyer, and A. Nagar, Phys. Rev. D 79, 064004 (2009).

[20] Y. Pan, A. Buonanno, R. Fujita, E. Racine, and H. Tagoshi, Phys. Rev. D 83, 064003 (2011).

[21] A. Buonanno, G. Faye, and T. Hinderer, Phys. Rev. D 87, 044009 (2013).

[22] A. Taracchini et al. (to be published).

[23] E. Barausse, A. Buonanno, S. A. Hughes, G. Khanna, S. O'Sullivan, and Y. Pan, Phys. Rev. D 85, 024046 (2012).

[24] E. Berti, V. Cardoso, and C. Will, Phys. Rev. D 73, 064030 (2006).

[25] E. Barausse, V. Morozova, and L. Rezzolla, Astrophys. J. 758, 63 (2012).

[26] E. Barausse and L. Rezzolla, Astrophys. J. 704, L40 (2009).

[27] Y. Pan et al., arXiv:1311.2565.

[28] T. Damour, A. Nagar, D. Pollney, and C. Reisswig, Phys. Rev. Lett. 108, 131101 (2012); A. Le Tiec, E. Barausse, and A. Buonanno, Phys. Rev. Lett. 108, 131103 (2012).

[29] http://www.black-holes.org/SpEC.html.

[30] C. Loken et al., J. Phys. Conf. Ser. 256, 012026 (2010). 